Revue de l'Institut des langues et cultures

d'Europe, Amérique, Afrique, Asie et Australie

$20 \mid 2014$

Le conte : d'un art à l'autre

\title{
Le conte et l'ironie romantique dans l'adaptation théâtrale du Chat botté par Ludwig Tieck
}

Folktale and Romantic Irony in Ludwig Tieck's Adaptation of Puss'n Boots for the Stage

Märchen und romantische Ironie in Ludwig Tiecks Umgestaltung des

Gestiefelten Katers für die Bühne

Jean-Charles Margotton

\section{OpenEdition}

\section{Journals}

Édition électronique

URL : http://journals.openedition.org/ilcea/2545

DOI : 10.4000/ilcea.2545

ISSN : 2101-0609

Éditeur

UGA Éditions/Université Grenoble Alpes

Édition imprimée

ISBN : 978-2-84310-290-5

ISSN : 1639-6073

Référence électronique

Jean-Charles Margotton, « Le conte et l'ironie romantique dans l'adaptation théâtrale du Chat botté par Ludwig Tieck », ILCEA [En ligne], 20 | 2014, mis en ligne le 08 décembre 2014, consulté le 01 mai 2019. URL : http://journals.openedition.org/ilcea/2545; DOI : 10.4000/ilcea.2545

Ce document a été généré automatiquement le 1 mai 2019.

(C) ILCEA 


\section{Le conte et l'ironie romantique dans l'adaptation théâtrale du Chat botté par Ludwig Tieck}

Folktale and Romantic Irony in Ludwig Tieck's Adaptation of Puss'n Boots for the Stage

Märchen und romantische Ironie in Ludwig Tiecks Umgestaltung des

Gestiefelten Katers für die Bühne

Jean-Charles Margotton

1 Lorsqu'en 1797, Ludwig Tieck fait paraître sa comédie Der gestiefelte Kater, il y a exactement cent ans qu'a été publié, dans les Contes de ma mère l'oye, le «Chat botté » de Charles Perrault. Le texte de Tieck fait partie des Volksmärchen von Peter Lebrecht, qui, entre autres, contiennent également Ritter Blaubart d'après La Barbe bleue de Perrault. Der gestiefelte Kater ${ }^{1}$ sera à nouveau publié par Tieck dans le troisième volume de son Phantasus, en 1812, c'est-à-dire à la date exacte de la parution de la première édition des Kinder- und Hausmärchen des frères Grimm. Mais la pièce du jeune Ludwig Tieck n'est pas intéressante seulement à cause de ces jolies coïncidences. Il faut souligner d'abord l'étonnante fécondité littéraire de Tieck qui, non seulement eut une très longue carrière, mais commença très tôt à publier des textes, avant même le début de ses études qu'il interrompit d'ailleurs, au bout de deux ans, pour se consacrer totalement à l'écriture. L'année 1797 fait partie des toutes premières du jeune romantisme allemand que parfois même on ne fait commencer que l'année suivante avec la fondation de la revue Athenäum à Iéna. Le texte que nous étudions illustre, avec d'autres, le passage, chez Tieck, d'un mode d'écriture encore marqué par l'influence de l'Aufklärung berlinoise et du goût littéraire dominant à un engagement sans ambages pour une orientation radicalement nouvelle dont il fut, sinon un pionnier, du moins un des premiers et plus féconds représentants. Le roman William Lovell précède d'un an notre texte et Franz Sternbald, qu'on considère comme un des grands romans du premier romantisme, en est le contemporain. Or s'il rencontra Friedrich Schlegel fin 1797, Tieck ne rejoignit le groupe 
d'Iéna que deux ans plus tard. Ajoutons, pour compléter ce cadrage rapide, un détail piquant: le recueil cité plus haut des Volksmärchen von Peter Lebrecht, où se trouve la première version de notre texte, avait été publié encore par Carl August Nicolai, c'est-àdire par le représentant le plus en vue du monde littéraire de l'Aufklärung. L'éditeur, faisant fond sur le succès d'une précédente publication sous ce pseudonyme, n'avait, pense-t-on, pas lu de très près les textes que le jeune auteur lui proposait. Toujours est-il que cette publication mena à la fin rapide de leur collaboration. Mais dans le même recueil en trois volumes, on trouve, comme autres reprises de sujets anciens, Ritter Blaubart qui transforme également le récit d'un conte en pièce de théatre, ainsi que les Schildbürger et des Volksbücher allemands du Moyen Âge. Le titre du recueil cherchait à l'évidence à faire pièce aux contes de Johann August Musäus publiés sous le titre Volksmärchen der Deutschen. Si ces textes s'inscrivent tous peu ou prou dans l'orientation critique dirigée contre la société contemporaine et sa conception de l'art, aucun ne va aussi loin, ne pousse la satire et le bouleversement des codes littéraires de manière aussi radicale que la comédie que nous étudions. Cette manière provocatrice et iconoclaste, Tieck la reprendra deux ans plus tard avec deux comédies : la pièce en six actes, Prinz Zerbino, qu'il écrivit en partie d'ailleurs en même temps que notre texte et dont le soustitre dit explicitement "Voyage au pays du bon goût, une sorte de suite du Chat botté », et Die verkehrte Welt («Le monde à l'envers »). Nous ne pourrons pas, bien sûr, aller au-delà de cette brève allusion. Pour terminer cette entrée en matière, il faut encore dire que le travail de Tieck à cette époque comporte une importante activité de traducteur, de découvreur littéraire, en particulier de l'œuvre de Shakespeare et de Gozzi, mais aussi, sans doute sous l'influence de son ami Wackenroder, des patrimoines allemand et européen des contes, légendes et Volksbücher, dont l'histoire de la Belle Maguelone, également de 1797.

2 Je me propose d'analyser la comédie satirique du Chat botté en ayant, bien entendu, comme ligne de mire, la question du passage d'un genre à un autre, à savoir de l'épique au dramatique. Simplement, cette question ne saurait être traitée sous le seul angle des changements formels, non seulement du fait de la complexité évidente ici du texte dramatique, mais aussi et surtout du fait de la portée programmatique de ces changements. À côté de la dimension formelle, il convient, en effet, de donner sa pleine importance à la dimension sémantique, quasi philosophique de la démarche de Tieck. Nous verrons donc d'abord l'organisation structurelle de la comédie en faisant ressortir la présence de trois niveaux de référence, de trois isotopies emboîtées, si l'on peut dire. Le premier niveau touche la question de la place du matériau narratif du conte repris de la tradition, première isotopie dans la construction complexe de l'ensemble. C'est pourtant par le deuxième niveau que nous commencerons, celui du jeu théâtral qui fait intervenir un public, un auteur, un bouffon et d'autres acteurs de l'entreprise dramaturgique. Nous placerons, au centre de ce niveau, et donc au centre du tout, la figure de l'auteur (fictif). Il faudra ensuite montrer qu'il existe un troisième niveau plus englobant, encore que moins immédiatement perceptible, mais essentiel pour la distanciation ironique qui caractérise l'entreprise. Nous nous proposons enfin de prolonger l'analyse par une réflexion sur la portée du texte, sa finalité polémique et esthétique, la place du merveilleux, enfin sur l'influence qu'il a eue.

3 La structure complexe de la pièce a été confirmée par Tieck lui-même lors de la seconde édition de son texte dans le recueil Phantasus : nous y reviendrons à l'occasion, mais les différences entre les deux versions sont plutôt de détail, comme dans la caractérisation 
de certains membres du "public ", ainsi que le note Tieck lui-même dans l'introduction aux Sämmtliche Werke ${ }^{2}$ (Tieck, 1841: III). En réalité, la différence essentielle, dans la seconde version, consiste dans l'encadrement des textes du recueil par un dialogue entre plusieurs amis dont certains lisent leurs textes aux autres qui les commentent ensuite. Tieck, le jeune Romantique, se place ainsi dans la lignée de Boccace et du Goethe des Entretiens des émigrés allemands, mais il annonce également Hoffmann et d'autres. Outre que ce paratexte constitue de fait un niveau supplémentaire dans la structure de la comédie comme texte global, il marque le souci de Tieck, alors même qu'il produit une œuvre en tous points surprenante et originale, de se placer sous l'égide de grands noms comme Aristophane ou Shakespeare ${ }^{3}$. Il est bien certain d'ailleurs qu'il aurait également pu se réclamer de la comédie italienne, de la libre créativité de Gozzi et de l'improvisation de la commedia dell' arte qu'il connaissait aussi fort bien. Quoi qu'il en soit, le «Prolog » qui ouvre la comédie, annonce d'emblée la couleur: ce qu'on va lire constitue un renversement des codes habituels, un bouleversement des perspectives: "La scène est dans le parterre ${ }^{4}$ ». C'est donc par la rupture de l'illusion théâtrale du fait de la suppression scandaleuse de la rampe, de la frontière entre le public et la fiction théâtrale, que Tieck choisit de commencer, nullement par l'évocation sans transition du conte de Perrault. Le dialogue introduit en effet un groupe de spectateurs dont les noms sont peut-être de simples indications de leurs professions (Fischer [Pêcheur], Müller [Meunier], Schlosser [Serrurier]...) et qui disputent librement, avant que le spectacle ne commence vraiment, de l'intérêt de l'œuvre qu'on veut leur montrer. Ce faisant, ils citent clairement le titre effectif de la comédie ("Un drôle de titre : Le Chat botté5 $»)$ et le fait que son contenu est annoncé comme un conte pour enfants (c'est bien ce que dit le sous-titre de l'œuvre : « ein Kindermärchen »). Ce n'est pas tout: leurs protestations, qu'ils formulent bruyamment avant même d'avoir vu quoi que ce soit et qui expriment leurs craintes que le spectacle ne corresponde pas à leur goût, finissent par attirer sur la scène l'auteur de la pièce luimême ("der Dichter ", p. 7) au moment justement où est prononcé le mot "goût " (les protestataires veulent sauver «le bon goût »). En jouant la carte d'une modestie quelque peu obséquieuse ( « je ne comprends pas moi-même comment j'ai pu avoir l'audace de me présenter devant vous $\left.{ }^{6} »\right)$, l'auteur réussit à retourner la situation et même à se faire applaudir par ces spectateurs versatiles. Mais cette victoire n'est que passagère. Jusqu'à la fin, la comédie sera entremêlée de retours aux réactions critiques du public, dont on a vite compris qu'il est composé de philistins, ce qui fait de notre texte une des premières expressions vivantes de la satire antiphilistine que le romantisme allemand se plut à exprimer en variations infinies, comme la face négative de son idéalisme. C'est évidemment surtout par cette transgression de la frontière entre spectacle et public que s'opère la distanciation satirique de l'illusion théâtrale. Le spectacle est, à chaque instant et pas seulement au cours des parties annexes (prologue, intermèdes et épilogue), son propre objet $^{7}$ : on pourra y voir une anticipation de formes plus modernes de distanciation, mais Tieck est conscient quant à lui de reprendre une forme de liberté esthétique qu'il trouvait déjà chez les auteurs du passé cités plus haut. Bien entendu, cette technique ironique ne remplace pas une situation stable (celle de l'illusion réaliste du théâtre habituel) par une autre qui, quoique dialectique, pourrait être le terme d'un processus. Le lecteur du texte, ou le spectateur réel dans le cas d'une représentation de l'œuvre, se voit tacitement invité à poursuivre la démarche, le texte de Tieck, malgré sa nature complexe, devenant à son tour un objet de thêâtre fictionnel presque comme un autre, susceptible, en tout cas, de susciter à son tour des prises de position critiques et donc d'être l'objet d'une distanciation. C'est d'ailleurs bien ce qui se passe dans le cadre 
de la publication dans le recueil Phantasus, avec la discussion entre les amis à propos des textes présentés par eux. On a le sentiment, inexact bien sûr, d'une ouverture infinie, celle qu'on a coutume de désigner par l'expression « mise en abyme ». Nous reviendrons plus tard sur la portée esthétique et philosophique de cette démarche. Disons simplement pour le moment qu'il ne s'agit pas pour le jeune Tieck d'un jeu gratuit pour se moquer d'un public philistin dont il sait bien qu'il est aussi le sien, il s'agit plus profondément de la réalisation littéraire de l'ironie romantique telle qu'elle sera définie un peu plus tard par Friedrich Schlegel et selon laquelle la démarche romantique de poétisation du réel ne peut se faire qu'au prix d'une remise en cause à chaque fois renouvelée de l'état auquel le sujet est parvenu, d'une succession incessante et en principe infinie d'autodestruction et d'autocréation. Le philistin se pense dépositaire d'un goût qu'il juge définitif et refuse à l'avance une création qui remettrait dangereusement en cause cet acquis rassurant. Or Tieck, au sujet duquel on ne fait sans doute pas fausse route en imaginant qu'il parle à travers le masque du "Poète ", ne vise pas tant cet infini que la défense d'un rapport plus naîf à l'art, l'enrichissement de la sphère poétique par la réintroduction d'éléments populaires précisément écartés par la critique et le goût de l'Aufklärung. Ce n'est pas un hasard si la comédie comporte côte à côte la reprise d'un conte pour enfants et le retour sur scène du bouffon, le Hanswurst, le personnage comique banni par Gottsched.

C'est donc sur cette isotopie, celle du spectacle lui-même comme lieu d'une confrontation entre l'auteur et son public, entre deux conceptions esthétiques divergentes, que Tieck choisit d'ouvrir et de fermer son texte. Ce spectacle, au demeurant, est d'une assez grande complexité. L'auteur est loin d'être le seul à être pris à partie par le public, c'est le cas du Hanswurst aussi. Par ailleurs, des intervenants habituellement confinés dans les coulisses, comme le machiniste ou le souffleur, paraissent également sur scène et prennent part à la diatribe qui, à plusieurs reprises, menace de faire échouer complètement l'entreprise. Notons encore que le spectacle est également une comédie-ballet; dès le prologue, une musique se fait entendre dont la fonction semble être d'abord de calmer les passions; cette musique est une citation explicite de la Flûte enchantée de Mozart. C'est grâce à elle que la paix revient à la fin du deuxième acte, le modérateur appelé à la rescousse par l'auteur se met dans la peau de Papageno et interprète la scène du glockenspiel, faisant pénétrer sur scène des animaux de toute sorte qui dansent au son des clochettes. Nous reviendrons sur la portée qu'il faut accorder à cette citation d'un opéra qui, notons-le, n'avait été donné pour la première fois que six ans auparavant, mais avait connu immédiatement un grand succès ${ }^{8}$. Il est remarquable que les spectateurs les plus sectaires se laissent séduire et apaiser par le ballet : pour Tieck, le Romantique, comme pour son ami Wackenroder et tous les autres, la musique, art suprême, est capable de transformer les âmes, même les plus philistines.

5 La reprise du conte de Perrault est donc, on l'a compris, insérée dans ce niveau complexe où la représentation théâtrale, si l'on peut dire, est son propre objet. C'est le conte qui lui donne un contenu plus traditionnel, bien que, comme on l'a dit, le jeu du conte soit régulièrement interrompu par l'intervention des «spectateurs » qui en font la critique. À propos de cette reprise du conte de fée comme sujet d'une comédie, nous allons tenter de montrer comment se fait le passage d'un genre littéraire à un autre, c'est-à-dire, d'abord, d'un mode d'écriture à un autre. En fait il est assez facile de montrer que la forme dialoguée n'est nullement absente du conte lui-même à l'origine : c'est le cas chez les prédécesseurs de Perrault, déjà, en particulier chez Basile dont on sent bien à quel point sa verve napolitaine se plait à donner aux propos des personnages (et surtout au père au 
début de l'histoire) un maximum de vie (Basile, 1634, 1674). Chez Straparola, qui avait déjà fait paraître en 1553 un conte sous le titre «La Chatte de Constantin le Fortuné », la part du dialogue direct est beaucoup moins grande, mais elle n'est nullement absente dans un récit qui se passe en Bohême et où le chat botté est une chatte-fée sans bottes. Chez Grimm, dont on sait que seule l'édition de 1812 contient l'histoire du Chat botté, il est remarquable que la forme dialoguée représente quasiment la moitié de la totalité du texte. La première venue du chat au château du roi, par exemple, est l'occasion d'une utilisation particulièrement plaisante du discours direct, avec le dialogue entre le chat, venu apporter des perdrix au roi, et les gardes qui ne veulent d'abord pas le laisser entrer 9. Même si Tieck a repris d'autres contes (comme «La Barbe bleue ", «Le Petit Poucet ", "Le Petit Chaperon rouge »), il semble que le conte du Chat botté ait déjà, en soi, une structure narrative qui donne une grande importance aux rencontres et aux échanges verbaux entre les personnages. Chez Perrault également le recours au style direct est incessant; et il faut y ajouter, même si la chose est moins sensible ici que dans d'autres récits de Perrault, que le ton même de la narration donne au lecteur fréquemment l'impression que le narrateur s'adresse directement à lui ${ }^{10}$ (Perrault, $\left.1994: 35\right)$. Le passage du récit à la forme dramatique chez Tieck entraîne évidemment une explosion de la partie dialoguée du texte, même s'il faut noter que cette forme n'est pas pour autant la seule dans le texte d'arrivée. En effet, les nombreuses didascalies sont, au sein du discours direct des personnages, comme un reste de l'élément proprement narratif initial, c'est-àdire proféré par un locuteur extérieur à l'histoire.

Il reste que la conséquence la plus visible de ce changement de genre est la multiplication des personnages et l'apparition d'épisodes totalement originaux. Pour faire ressortir cet aspect, il convient de dire d'abord que Tieck a repris assez fidèlement le canevas du conte de Perrault. La comédie est en trois actes : le contenu du conte est réparti logiquement, sans bouleversement sensible, entre ces trois actes. Le premier présente la situation après la mort du pauvre paysan : les trois fils s'appellent Lorentz, Barthel et Gottlieb (qui sera le Marquis de Carabas). C'est Lorentz qui est chargé de faire au début le récit de la situation, le père n'apparaît pas et on est donc loin de Basile où ce père avait un rôle si original. Tieck garde la répartition à première vue injuste de l'héritage, le désespoir du plus jeune fils (Gottlieb, au nom sans doute prometteur, puisqu'il signifie Amédée, aimé de Dieu, donc), les propositions du chat (qui s'appelle «Hinze », c'est-à-dire approximativement "Tartempion») et la fabrication des bottes (le cordonnier est ici un vrai personnage secondaire qui appelle le chat "Musje », c'est-à-dire « Monsieur »). Dans le même acte, on a ensuite l'introduction du roi et de sa fille (la princesse a jusqu'ici refusé tous les prétendants, comme dans le conte «König Drosselbart $\left.{ }^{11} »\right)$, enfin l'allusion à l'ogre qui est ici un magicien croque-mitaine ("Popanz», les Grimm diront "Zauberer », c'est-à-dire " magicien ») au cours d'une scène originale dans une auberge de campagne.

7 Le deuxième acte contient les épisodes du chat chasseur, très proches de Perrault, mais l'expansion de la matière événementielle est rendue plus sensible par l'introduction de nouveaux personnages, comme le bouffon du roi ou l'érudit de la cour dans des scènes très développées, ou encore le couple d'amoureux stéréotypés ( $\mathrm{Er} / \mathrm{Sie})$, dont les effusions, empruntées à la littérature sentimentale à la mode, viennent troubler la chasse du chat. Ce couple reviendra au troisième acte, mais ce sera pour montrer au grand jour son déchirement. $\mathrm{Si}$, dans ce dernier acte, Tieck reprend bien, de manière très condensée, les épisodes restants du conte (la baignade, les menaces adressées aux paysans permettant d'officialiser le titre du Marquis, la visite au croque-mitaine avec le motif de 
la métamorphose de ce dernier en divers animaux, en particulier en souris, ce qui entraîne sa perte, enfin la montée en grade de Gottlieb, son mariage avec la princesse et l'anoblissement du chat Hinze), ici ce contenu narratif disparaît presque sous le foisonnement des éléments annexes et parasitaires. Le bouffon, des courtisans, envahissent l'espace scénique et se mêlent aux techniciens du spectacle, et l'accession du faux marquis à la dignité royale se fait par la reprise explicite des épreuves finales de Tamino dans La Flûte enchantée. À la fin, la frontière entre l'isotopie du conte et celle du spectacle est devenue très floue. Déjà, au premier acte, la confusion des niveaux avait été amorcée fugitivement lorsque le roi s'était étonné que le prince Nathanael, le prétendant à la main de sa fille, censé régner sur un pays très lointain, parle la même langue que lui :

Encore une chose, dites-moi un peu comment il se fait que vous, qui habitez si loin, parliez si couramment notre langue? - Silence!-Comment?-Silence, silence ! - Je ne comprends pas - (lui parlant à voix basse) Calmez-vous, sinon le public, là en bas, va finir par s'apercevoir que cela n'a rien de naturel ${ }^{12}$. (Tieck, 1964 : 20)

Cet échange fait partie des éléments par lequel s'opère la rupture de l'illusion théâtrale. Mais, au sein même des épisodes du conte, on note un élément semblable : Gottlieb, le fils désavantagé, vient de se répandre en plaintes sur son sort injuste; le chat Hinze intervient alors : « Hinze, le chat (se relève, s'étire, fait le dos rond, baille, puis dit) - Mon cher Gottlieb, - vous me faites vraiment pitié. [...] Gottlieb (étonné) - comment, chat, mais tu parles $^{13}$ ?» (Tieck, 1964 : 11) Rien de tel chez Perrault où la capacité du chat à parler n'est pas autrement relevée, ni ressenti comme curieux son désir de se faire confectionner des bottes. Chez Grimm, certes, le fils du meunier s'étonne («Le fils du meunier s'étonna que le chat parlât ainsi $\left.{ }^{14} »\right)$, mais cela n'entraîne aucun changement de point de vue, pas davantage en tout cas que l'incise, un peu plus tôt, quand le chat répond aux gémissements de son maître ("Écoute, commença le chat, qui avait tout compris...»). Dans le cas de la comédie de Tieck, au contraire, et cette remarque vaut pour l'ensemble de la reprise du conte ici, c'est, avec la remarque étonnée de Gottlieb, la dimension merveilleuse du conte qui, d'un coup, disparaît. À la place du merveilleux qui, traditionnellement, abolit, par une entente tacite entre narrateur et lecteur, la distance entre le réel et l'imaginaire, c'est ici la fantaisie et l'ironie qui coloreront l'évocation de l'histoire du chat et de son maitre. Tieck prend plaisir à jouer avec la frontière entre les deux univers : le chat, devenu chasseur, se met par exemple à ronronner, ou à griffer. En un mot, la distanciation qui caractérisait le niveau du spectacle que nous avons évoqué d'abord se retrouve également au cœur même de la comédie, dans l'histoire du Chat botté. Du coup, le dialogue peut devenir tout autre chose que le vecteur des événements, on voit le roi faire un tableau bien négatif du mariage, la princesse est un bas-bleu qui se pique de sciences et de littérature, on se croit parfois même dans le Léonce et Léna de Büchner quand il est question de la taille minuscule des États qui entourent le royaume, lui-même bien exigu, du roi, à l'image de la Kleinstaaterei allemande dont Büchner fait la satire. Dans son adaptation assez fidèle de l'œuvre de Tieck, Tankred Dorst a pu utiliser facilement le canevas originel pour faire allusion à des événements et phénomènes plus modernes (Dorst, 1964). La structure comique choisie permet, en effet, comme par exemple dans la tradition viennoise d'un Nestroy, de rattacher un contenu satirique, politique, moral ou autre, à une trame comique ou féérique traditionnelle. Tieck avait particulièrement été sensibilisé à cette attitude distanciée par ses études sur Shakespeare qui l'avaient conduit, dès 1793, à rédiger un essai, Shakespeares Behandlung des Wunderbaren. Il est clair donc que le respect de la source n'est pas l'essentiel. Bien plus 
important pour lui est le plaisir du jeu (Scherz) opposé au sérieux (Ernst) de la société philistine. On le voit bien, le public est constamment déstabilisé, dérouté, provoqué par le mélange des niveaux dans le spectacle qui lui est proposé.

Avant d'aborder la question de la finalité de cette reprise libre du conte, essayons de voir quels changements structurels elle entraîne. Il est assez aisé, à l'aide des concepts de l'étude morphologique des contes (Propp / Greimas), de décrire le conte originel, celui de Perrault, comme suit: la situation de départ est caractérisée par le désordre que représente l'injustice apparente qui frappe, dans la répartition de l'héritage du pauvre meunier, le plus jeune des fils. Cette injustice objective (au moins dans une première approche du sujet) n'est d'ailleurs que le reflet, dans le monde des pauvres gens, du système féodal de transmission du pouvoir par primogéniture. Le fait qu'il y ait trois fils (ce n'est pas le cas chez Basile qui ne parle que d'un seul frère de Gagliuso) peut être interprété comme une influence de la structure quasi systématiquement tripartite des contes. Quoi qu'il en soit, le conte lui-même, comme récit, est l'exposé de l'action qui conduit à la réparation de cette injustice initiale, de ce «manque ». Le " héros » est, à l'évidence, le fils du meunier qui aspire à sortir de sa misère et est en quête de fortune, de pouvoir et d'amour. Toutes choses qu'il obtiendra grâce à l'intervention " merveilleuse " du chat, que nous devons considérer comme "adjuvant». Le rôle de ce dernier est cependant tellement déterminant et donne à ce point sa coloration particulière au conte tout entier qu'il n'est pas étonnant qu'il devienne même le personnage-titre (ce n'est pas le cas chez Basile). En face de cet adjuvant, l'ogre (chez Perrault) ou le magicien (chez Grimm) est l'«opposant » qui cherche à se mettre en travers de la quête du héros. Le conte est un récit optimiste dans la mesure où l'adjuvant l'emporte facilement par ses ruses sur les pouvoirs magiques de l'opposant. Le héros parvient à atteindre l'« objet » de sa quête : le trône, la princesse, un statut social nouveau et supérieur. Le nouveau roi, à la fin du récit, est ainsi le « destinataire » clair de l'entreprise. Quant au dernier actant du système, le "destinateur ", il me semble qu'il est possible de l'identifier comme un actant abstrait, non clairement incarné par un personnage, mais néanmoins à l'œuvre dans l'évolution même du récit, dans sa logique interne, à savoir la justice immanente, le principe moral qui exige de corriger le désordre initial que constituait la situation du pauvre héritier. Dans le cas particulier de ce conte, cet actant, toujours plus ou moins assimilable à un Dieu implicite, une Providence, est d'une nature assez ambivalente, sa moralité assez douteuse : certes, l'injustice initiale est claire, mais, d'une part, l'héritage se révèle beaucoup moins décevant qu'il n'y paraît d'abord et surtout, les moyens mis en œuvre par le chat, ses ruses, ses duperies, à commencer par l'air de noblesse qu'il se donne grâce aux bottes, tout cela jette une ombre sur la légitimité de l'entreprise ellemême. Il n'est guère douteux que cette ombre soit la raison même du succès de cette histoire et sans doute aussi la raison qui amena Tieck à la choisir pour sa propre entreprise.

10 On peut s'amuser, pour compléter l'analyse, à voir comment le schéma des actants pourrait fonctionner chez Tieck, si l'on considère, dans la comédie, non seulement le conte lui-même, qu'il expose sous forme dialoguée (le schéma qu'on vient de présenter y serait d'ailleurs tout à fait valable), mais la totalité du texte avec ses différents niveaux. Le "héros» est ici, me semble-t-il, l'auteur (Der Dichter) dans son débat avec le Public. L'« objet » de sa quête est le changement de paradigme auquel le romantisme aspire dans le domaine de la poésie, de l'art et même de la totalité de l'existence. C'est ce que dit l'auteur à la fin de la pièce, s'adressant au public après avoir constaté l'échec (au moins 
apparent) de son entreprise : «Il aurait fallu que vous redeveniez des enfants ${ }^{15}$ » (Tieck, 1964 : 62). L'« adjuvant ", ce sont ici les moyens mis en œuvre par l'auteur, le traitement scénique du conte, la satire, l'ironie, la création de nouveaux personnages, la place de la musique. Or, il est clair que l'«opposant» est plus fort: à la fin du spectacle, le philistinisme du public n'a pas été surmonté par le jeu théâtral proposé. S'il est assez facile de voir dans l'auteur réel, Tieck, le «destinateur» du système, celui qui, en coulisses (derrière les fausses coulisses de la comédie), tire les ficelles, à savoir le Romantique aspirant à "romantiser » le monde, le «destinataire » ici reste hautement indistinct et problématique : ce serait le public idéal, le spectateur capable d'enrichir son savoir et sa raison par la fantaisie et la naïveté préservée de son enfance et qui pourrait, dans la démarche dialectique de l'ironie romantique, poursuivre à son tour le processus infini de poétisation du réel. C'est ce que nous, lecteurs réels de Tieck, nous pourrions penser être, si cette simple supposition n'était pas déjà la preuve que nous ne le sommes point, hélas! Consolons-nous en pensant que, si l'œuvre de Tieck a bel et bien eu une suite et une postérité (j'ai évoqué tout à l'heure Tankred Dorst), c'est bien que l'échec que vit l'auteur dans la comédie à la fin de celle-ci n'est qu'apparent et que le grain a germé.

11 Pour finir, il n'est pas sans intérêt de se demander quelles raisons ont conduit Tieck à choisir le conte de Perrault comme matériau dans son entreprise de déconstruction ironique. Le fait qu'il s'agisse d'un conte pour enfants est essentiel, bien entendu, l'auteur l'avoue clairement à la fin en s'adressant au public qui siffle sa pièce : «J'avais tenté de vous ramener aux impressions lointaines de vos années d'enfance pour que vous receviez le conte représenté ici sans le prendre pour plus important qu'il n'est ${ }^{16}{ }^{\prime}$ (Tieck, 1964 : 61 et suiv.). Hélas! Le public n'a pas réussi à oublier un moment sa culture et son statut d'adulte. La citation de certains éléments de La Flûte enchantée de Mozart va dans ce sens : Tieck a choisi, dans l'opéra, d'une part la fantaisie naïve, mais triomphante de Papageno et de son glockenspiel, mais aussi le thème maçonnique de l'initiation, des épreuves auxquelles le héros doit se soumettre avant d'accéder à la véritable humanité. Sans doute y a-t-il un peu d'amertume dans sa démarche puisque le public de la comédie (comme le public réel en Europe) tombe sous le charme de la musique alors qu'il reste insensible aux tentatives éducatives de l'auteur.

Devons-nous rechercher d'autres raisons pour expliquer le fait que Tieck ait choisi précisément le conte du Chat botté? Les allusions à la coloration potentiellement révolutionnaire du sujet ${ }^{17}$ sont évidentes, Tieck interprète ici ou là le conte du pauvre homme devenu roi à la lumière de la récente Révolution française. Mais il ne convient pas, à notre avis, de solliciter davantage cet aspect qui, bien plutôt, fait partie de la stratégie ironique et satirique de l'œuvre. Plus intéressant semble être le fait que l'histoire de Perrault est celle d'un changement de statut et d'identité, de l'accession d'un être que la vie n'avait pas «gâté » à une existence supérieure, ce qu'on peut lire comme une allégorie de l'utopie romantique. Surtout, le chat prend aussi dans le dispositif de Tieck une place centrale : ses ruses, son déguisement (ses bottes ne sont pas, notons-le, les bottes de sept lieues du Petit Poucet, elles sont inutiles si elles ne sont la marque apparente d'un statut social aristocratique), sa faconde, toutes choses qui sont déjà chez Perrault, mais que Tieck a considérablement amplifiées, le désignent à l'évidence non seulement comme le trickster qui dupe efficacement son monde, mais, dans la logique intratextuelle, comme l'acteur en soi, le représentant symbolique du théatre, l'incarnation de la recherche romantique d'une action de l'art sur le monde ${ }^{18}$. 


\section{BIBLIOGRAPHIE}

BASILE Giambattista (1634), « Gagliuso », Lo cunto de li cunti, puis Il Pentamerone.

BASILE Giambattista (1674), Il Pentamerone.

BASILE Giambattista, Le Conte des contes ou Le Divertissement des petits enfants (trad. Françoise

Decroisette), Strasbourg : Circé, 1995.

DORST Tankred (1986), Der Kater, oder Wie man das Spiel spielt, nach Ludwig Tieck (1964), Werkausgabe vol. 3, Frühe Stücke, Berlin : Suhrkamp.

GRIMM Brüder (1812), Kinder- und Haus-Märchen, Berlin.

GRIMM Jacob et Wilhelm, Les contes. Kinder- und Hausmärchen (2 vol.), texte français et présentation par Armel Guerne, Paris : Flammarion, 1967.

PERRAult Charles, Contes de ma Mère l'Oye, Paris : EJL, Librio, 1994.

RIMASSON-FERTIN Natacha (2009), Les Frères Grimm, Contes pour les enfants et la maison (2 vol.)

collectés par les frères Grimm, édités et traduits par Natacha Rimasson-Fertin, Paris : éditions José Corti.

STRAPAROLA Giovanni Francesco, « La chatte de Constantin le Fortuné », Les Nuits facétieuses (Jean Louveau et Pierre de Larivey, trad.), revue, corrigée et commentée par Joël Gayraud, Paris : éditions José Corti, 1999.

STRAPAROLA Giovanni Francesco (1550-1553), Le piacevoli notti.

TIECK Ludwig (1964), Der gestiefelte Kater, Stuttgart : Reclam, 2011 (rééd.).

TIECK Ludwig (1841), Sämmtliche Werke (1 ${ }^{\mathrm{er}}$ vol.), Paris : Baudry’s europäische Buchhandlung.

\section{NOTES}

1. Dont la création n'aura lieu qu'en avril 1844 à Berlin.

2. Tieck désigne ici sa pièce comme «farce » («Schwank») ou " opuscule » («Werkchen ») et se défend d'avoir voulu réaliser une satire ad hominem. Il critique ceux qui ne comprennent la plaisanterie («Scherz, Laune und Witz») (Tieck, 1841: IV) que sous une forme prosaïque et personnelle.

3. Voir « Völliger Schluss» à la suite de la lecture du Gestiefelter Kater dans Phantasus (Tieck, 1841 : 489 et suiv.).

4. « Die Szene ist im Parterre» (Tieck, 1964 : 5). Nous donnerons désormais le $\mathrm{n}^{\circ}$ de la page de cette édition directement après les citations. Les traductions sont de l'auteur de cet article.

5. « Ein wunderlicher Titel ist es: der gestiefelte Kater.» (Tieck, 1964 : 6)

6. " [ich] begreife selbst nicht, wie ich zu der Kühnheit komme, so vor Ihnen zu erscheinen. " (Tieck, $1964: 8)$

7. " Fischer: Sagt mir nur, wie das ist, - das Stück selbst, - das kömmt wieder als Stück im Stück vor. » («Pêcheur : Expliquez-moi un peu cela - la pièce elle-même - est présentée comme pièce dans la pièce. ») (Tieck, $1964: 50)$ 
8. Dès 1794 , la mère de Goethe avait attiré l'attention de l'écrivain sur ce spectacle auquel elle avait assisté à Francfort, en soulignant avec enthousiasme son succès incroyable auprès du public (d'ailleurs, elle avait surtout apprécié la scène que Tieck retient précisément, celle du glockenspiel qui apaise Monostatos et les animaux).

9. « Die Wache rief: 'Halt! Wohin?' - 'Zum König!' antwortete der Kater kurzweg. 'Bist du toll, ein Kater und zum König?' - 'Laß ihn nur gehen', sagte ein anderer, 'der König hat doch oft Langeweile, vielleicht macht ihm der Kater mit seinem Brummen und Spinnen Vergnügen.' ( "Le garde s'écria: Halte ! Où vas-tu ? - Voir le roi ! répondit le chat sans détour. - As-tu perdu la tête, un chat qui veut voir le roi ? - Laisse-le passer, dit un autre, souvent le roi s'ennuie, peut-être que le chat le distraira avec ses ronronnements. ») (<www.1000-maerchen.de/fairyTale/852-der-gestiefelte-kater.htm>) 10. Par ex. : «[...] il gagna aussitôt les gouttières, non sans peine et sans péril, à cause de ses bottes qui ne valaient rien pour marcher sur les tuiles. »

11. Dans la traduction d'Armel Guerne, ce conte s'intitule «Le roi Barbabec " (Guerne, 1967, vol. 1 : 293-298) ; dans celle de Natacha Rimasson-Fertin, il s'agit du conte « Le roi Bec-de-grive » (Rimasson-Fertin, 2009, vol. 1 : 289-294).

12. "Aber noch eins, sagen Sie nur, da Sie so weit weg wohnen, wie Sie unsere Sprache so geläufig sprechen können? - Still! - Wie? - Still! Still! - Ich versteh nicht - (leise zu ihm) Seien Sie doch ja damit ruhig, denn sonst merkt es ja am Ende das Publikum da unten, dass das eben sehr unnatürlich ist. »

13. " Hinze, der Kater (richtet sich auf, dehnt sich, macht einen hohen Buckel, gähnt und spricht dann) - Mein lieber Gottlieb, - ich habe ein ordentliches Mitleid mit euch. [...] Gottlieb (erstaunt) - Wie, Kater, du sprichst?»

14. « Der Müllersohn verwunderte sich, daß der Kater so sprach. "

15. «Sie hätten wieder zu Kindern werden müssen. »

16. "Ich hatte den Versuch gemacht, Sie alle in die entfernten Empfindungen Ihrer Kinderjahre zurückzuversetzen, dass Sie so das dargestellte Märchen empfunden hätten, ohne es doch für etwas Wichtigeres zu halten, als es sein sollte. »

17. « Ein Revolutionsstück, so viel ich begreife » («Une pièce révolutionnaire, si je ne m'abuse »), dit un spectateur d'emblée (Tieck, $1964: 6$ ) et Hinze, le chat, s'exclame lui-même, au moment où il vient d'éliminer le croque-mitaine, incarnation d'une Loi tyrannique et injuste: «Freiheit und Gleichheit! - das Gesetz ist ausgefressen! Nun wird ja wohl der Tiers État Gottlieb zur Regierung kommen. » («Liberté et égalité ! On a mangé la Loi ! Désormais Gottlieb, le Tiers État va prendre le pouvoir, je pense.» (Tieck, $1964: 58)$

18. On peut voir une confirmation de cette interprétation dans le fait que Tieck a introduit dans son dispositif le personnage du bouffon, également lié au monde du théâtre, et lui a donné une place très importante au côté du chat et comme son double.

\section{RÉSUMÉS}

La première version du Chat botté de Ludwig Tieck parut en 1797 dans un recueil de textes (qui comportait également une adaptation de Barbe bleue) publié par un éditeur, Friedrich Nicolai, qui était une figure importante de l'Aufklärung berlinoise. On peut se représenter la pièce comme un pont entre le monde de pensée des Lumières et celui du Romantisme. Il s'agit de montrer non seulement comment l'œuvre "déconstruit» le conte de Perrault en l'intégrant dans un jeu théâtral entièrement fait de ruptures, mais en quoi le récit du conte est plus que le fil rouge du 
drame. Il y a en effet une correspondance entre la conception de l'univers poétique du conte comme univers de l'imaginaire enfantin autosuffisant et la conception romantique nouvelle de la poésie et de l'art comme sphères autonomes, en quoi la satire qui structure toute l'œuvre se manifeste comme différente de celle que pratique la critique rationaliste : elle n'a d'autre cible que le refus d'admettre l'art comme lieu de la liberté absolue.

The first version of Ludwig Tieck's Puss'n Boots appeared in 1797, in a collection of works that also included his adaptation of Bluebeard and that was published by Friedrich Nicolai, an important figure in the Berlin Aufklärung. The play can be seen as a bridge between the worldviews of the Enlightenment and Romanticism. The question is to show not only how the work 'deconstructs' Perrault's narrative, by casting it in a theatrical game made entirely of ruptures, but also how storytelling here is more than the story line. There is, in fact, a connection between the conception of the story's poetic universe as the self-contained child-like imagination and the new romantic conception of poetry and art as autonomous spheres. As such, the satire that structures the whole work proves to be different from a rational critique: its target is none other than the refusal to accept art as the space of absolute liberty.

Die 1797 vom jungen Ludwig Tieck veröffentlichte Umgestaltung des Märchens „Der Gestiefelte Kater" als Teil der Volksmärchen des Peter Lebrecht gehört zu den allerersten Texten der jungen romantischen Schule in Deutschland. Hier wird die Erzählung, die er bei den Italienern und bei Perrault gefunden hatte, zu einer Komödie. Doch hat der Übergang von der Epik zur Dramatik bei diesem Verehrer von Shakespeare und der commedia dell'arte viel mehr zur Folge als nur eine Änderung der literarischen Form. Alle normalerweise getrennten Ebenen - Geschichte, Schauspiel, Figuren, Autor, Publikum, ja sogar Bühnentechniker - werden durcheinander gemischt, es erfolgt eine verblüffende ironische Verfremdung des märchenhaften Stoffes. Jedoch ist der entscheidende Punkt dieser auf das Publikum gerichteten Ironie, die ein erstes Beispiel der romantischen Philisterkritik darstellt, folgender: Die Welt der Poesie soll durch die Wiedereinführung gerade der volkstümlichen Elemente bereichert werden, die die Kritik und der literarische Geschmack der Aufklärung abgelehnt hatten.

\section{INDEX}

Mots-clés : romantisme, comédie romantique, conte populaire, réécriture, philistin Schlüsselwörter : Romantik, romantische Komödie, Volksmärchen, Umgestaltung, Philister Keywords : romantic, romantic comedy, fairy tale, rewriting, philistine

\section{AUTEUR}

\section{JEAN-CHARLES MARGOTTON}

Professeur émérite, Université Lumière-Lyon 2 